\title{
Surface EMG Signal Amplification and Filtering
}

\author{
Jingpeng Wang \\ School of Engineering and \\ Advanced Technology \\ Massey, New Zealand
}

\author{
Liqiong Tang \\ School of Engineering and \\ Advanced Technology \\ Massey, New Zealand
}

\author{
John E Bronlund \\ School of Engineering and \\ Advanced Technology \\ Massey, New Zealand
}

\begin{abstract}
Electromyographic (EMG) signals have been widely employed as a control signal in rehabilitation and a means of diagnosis in health care. Signal amplification and filtering is the first step in surface EMG signal processing and application systems. The characteristics of the amplifiers and filters determine the quality of EMG signals. Up until now, searching for better amplification and filtering circuit design that is able to accurately capture the features of surface EMG signals for the intended applications is still a challenging. With the fast development in computer sciences and technologies, EMG signals are expected to be used and integrated within small or even tiny intelligent, automatic, robotic, and mechatronics systems. This research focused on small size amplification and filtering circuit design for processing surface EMG signals from an upper limb and aimed to fix the amplifiers and filters inside a robotic hand with limited space to command and control the robot hand movement. The research made a study on the commonly used methodologies for EMG signal processing and circuitry design and proposed a circuit design for EMG signal amplification and filtering. High-pass filters including secondorder and fourth-order with the suppression to low frequency noises are studied. The analysis, verification and experiment showed that a second-order high-pass filter can adequately suppress the low frequency noises. The proposed amplification and filtering circuit design is able to effectively clean the noises and collect the useful surface EMG signals from an upper limb. The experiment also clearly revealed that power line interference needs to be carefully handled for higher signalnoise-ratio (SNR) as a notch-filter might cause the loss of useful signal components. Commercial computer software such as LabView and Matlab were used for data acquisition software development and data analysis.
\end{abstract}

\section{General Terms}

EMG data acquisition, Bio-signal processing, Bio-informatics

\section{Keywords}

EMG signals, data acquisition, signal processing.

\section{INTRODUCTION}

EMG signal is the electrical expression caused by neuromuscular activation during muscular contraction, depicting the current detected at the specific location that is produced by the ionic flow across muscle fiber membranes and transmitted through intervening tissues. The motor unit is the most elementary functional unit of a muscle, generating a motor unit action potential (MUAP) when activated. Repeated continuous activation of motor units generates motor unit action potential trains (MUAPT) that are superimposed to form the EMG signal [1].
Collecting electromyographic (EMG) signals emanated from the human body using electrodes has become a routine procedure both in rehabilitation engineering and medical research. The concept of myoelectric control to manipulate an artificial hand using EMG signals from contracting muscles was proposed in the late 1940s and the great progress has occurred since the early 1960s. Nowadays, different types of assistive devices such as externally powered prostheses and electrically powered wheelchairs are controlled using EMG signals.

EMG signal is fairly weak and with the unwanted noises. It has to be carefully processed before using it for any health care applications. Signal amplification and filtering plays a critical role in EMG practical application systems. The process is expected to remain as much information from the physiological EMG signals as possible while it minimises the noise components. The two major functions of amplification and filtering process are the maximisation of the signal to noise ratio and the minimisation of the distortion of the signal. Although there have been many reports and approaches about the design of amplifiers and filters for EMG signal acquisition, better circuit structure and optimised design are still the focus of further research for accurate and reliable EMG signal driven systems.

This paper presents a design of an EMG signal amplification and filtering circuit using an instrumentation amplifier with differential amplification technology and several active filters with Sallen-Key architecture. The order of high-pass and lowpass filters in the proposed circuit and the sampling rate that is appropriate to the analog-to-digital conversion of EMG signals were investigated. LabView together with the hardware from National Instrument and the electrodes from Thought Technology Ltd were employed for capturing EMG signals and the testing of the circuits. Matlab was used for data analysis and comparison. The expected performance of the amplification and filtering circuit has a good match with the analysis and the experiment result.

\section{EMG SIGNAL AND THE NOISES}

It is well accepted that the amplitude of the EMG signal is within a range from the $\mu \mathrm{V}$ to low $\mathrm{mV}(0-6 \mathrm{mV}$ peak-to-peak or 0-1.5 mV RMS). The energetic distribution of EMG signal is basically within the 0 to $500 \mathrm{~Hz}$ range in frequency domain, with the dominant components in the $50-150 \mathrm{~Hz}$ range. Outside the $0-500 \mathrm{~Hz}$ frequency range, signals with energy less than electrical noise level are unusable. Four primary types of noise sources contribute to the process of EMG signal acquisition, i.e. the inherent noise of electronic parts inside the signal detection and recording instrument, the ambient noise from the electromagnetic radiation in the environment, the motion artefacts with electrical signals mainly in the frequency $0-20 \mathrm{~Hz}$ range from the electrode-skin interface and from movement of the cable connecting the electrode to the amplifier, and the inherent instability of the EMG signal with unstable 
components in $0-20 \mathrm{~Hz}$ range due to the quasi-random nature of the firing rate of the muscular motor units [2].

The property of amplitude, time and frequency of EMG signals is influenced by the anatomical and physiological characteristics of muscles, the control mechanism of the nervous system, and the instrumentation involved. More accurately, multiple factors determine the property of EMG signals such as the timing and intensity of muscle contraction, the distance from the electrode to the target muscle, the amount of adipose tissue between the skin and the muscle, the properties of the electrode and the amplifier, and the contact between the skin and the electrode [3].

\section{AMPLIFICATION AND FILTERING CIRCUITRY}

The quality of an EMG signal from the electrodes is partially dependent on the properties of the amplifiers. Because of the weak amplitude of EMG signals typically in the order of tens to thousands $\mu \mathrm{V}$, it is necessary that the gain of the amplifiers used in EMG applications is in the range from 1000 to 10000. Consequently, the amplification process commonly incorporates several stages. Figure 1 illustrates the block diagram of the amplification and filtering stages implemented in the circuit presented in this paper. The most important stage, namely the first stage close to the electrodes, is conventionally called pre-amplifier. The consideration to incorporate a preamplifier is to have a high common mode rejection ratio (CMRR), a high input impedance, a short distance to the signal source, and a strong direct current (DC) signal suppression [3]. The outcome from the pre-amplifier is then processed by a high-pass and a low-pass filter before entering into the second amplification stage that amplifies signals again to attain an expected gain. The second amplification stage is a simple inverting amplifier and its gain can easily be adjusted by choosing different resistors. To further suppress high frequency noises, a low-pass filter follows the second amplification stage. Finally, the output signal from the amplification and filtering circuit is fed into an analog-digital converter (ADC).

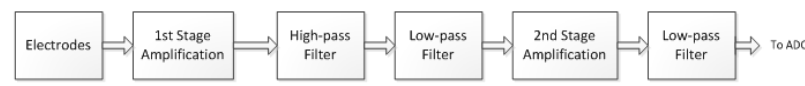

Fig. 1: Block diagram of amplification and filtering circuitry

\subsection{The Electrodes}

The design of the electrode is of paramount importance as the quality and the design of the electrodes directly affect the quality of the EMG signals. There are mainly two types of electrodes on the market, non-invasive surface electrode and invasive inserted electrode (wire or needle). Both have been used for EMG signal detection. The surface electrode has two categories, passive and active. The passive electrode has a conductive (commonly metal) detection surface touching with the skin, whereas the active electrode has a differential amplifier with a passive electrode in order to reduce the effects of capacitance coupling and provide very low output impedance, rather than transmitting the signal to the amplifier through long lead wires. The advantage of active electrode is the reduced capacitance coupling and the very low output impedance will not introduce significant noises from power line and cable movement artefact, but such electrodes are expensive and complicated $[1,3]$. Surface electrodes were selected for this research as they are non-invasive and economic.

\subsection{Pre-amplifier}

The pre-amplifier with a reference-driven and a shieldingdriven circuit has an instrumentation amplifier INA128 and an operational amplifier OPA2604 both from Texas Instruments. INA128 is a low power, general purpose instrumentation amplifier offering excellent accuracy with very low offset voltage, drift and high common-mode rejection. The gain is adjustable from 1 to 10,000 through equation (1):

$$
G=1+\frac{50 k \Omega}{R_{G}}
$$

where $R_{G}$ is an external resistor [4].

As showed in Figure 2, the EMG signal from the electrodes is fed into the positive and negative input pins of INA128. $R_{1}$ and $R_{2}$ comprise the resister $R_{G}$. A signal from the mid-point of $R_{1}$ and $R_{2}$ is fed into OPA2604 to provide a guarding potential and a reference potential.

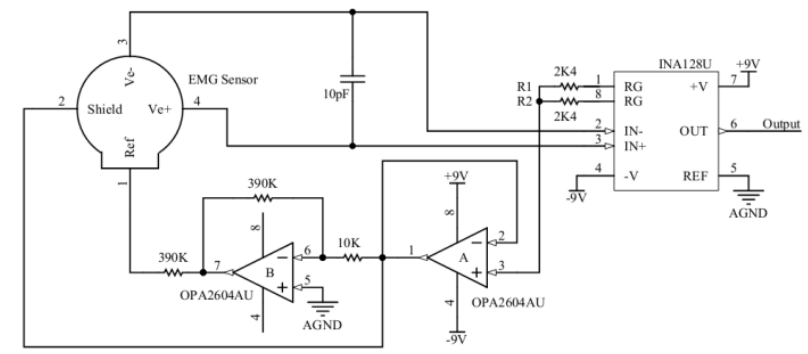

Fig.2: The schematic circuit of the pre-amplifier with reference and shielding-driven

Because of the weakness of the EMG signal, the difference in the amplitude between some useful EMG signal components and the noises can be very small. If the gain of the pre-amplifier is set too large, the noises will be simultaneously amplified enormously, thus leading to the instability and saturation of the subsequent amplifier. To avoid such a consequence, the gain of the pre-amplifier is preferred to be set around 10. For the preamplifier designed for this research, the gain was calculated using equation (1) and set at 11.4.

$$
G_{1}=1+\frac{50 k \Omega}{R_{G}}=1+\frac{50 k \Omega}{R_{1}+R_{2}}=11.4
$$

where $G_{1}$ is the gain of the pre-amplifier; both $R_{1}$ and $R_{2}$ are $2.4 \mathrm{k} \Omega$ resisters.

\section{HIGH-PASS AND LOW-PASS FILTER}

In the amplification and filtering circuitry, high-pass filters and low pass-filters were used after the first and second amplification stages. The reason is that the noises and the EMG signals are simultaneously amplified, which is not favourable for the following process. To design a filter, the corner frequency, the roll-off rate, and the circuit topology have to be chosen. The order of a filter determines the roll-off rate of the filter, namely the slope of its frequency response curve. A firstorder filter has a roll-off rate of $-6 \mathrm{~dB} /$ Octave whereas a secondorder filter has $-12 \mathrm{~dB} /$ Octave, that is, the roll-off rate of the filter response is proportional to the order of a filter. Higherorder filters are usually constructed by cascading first- and second-order blocks [5].

There are several types of noises with different characteristics within the low frequency components. As a result, the design a 
high-pass filter is more complicated comparing with low-pass filter design. In recent years, more research has been seen in high-pass filter design and different corner frequencies including $5 \mathrm{~Hz}, 10 \mathrm{~Hz}, 10-20 \mathrm{~Hz}, 20 \mathrm{~Hz}$, and $15-28 \mathrm{~Hz}$, and different roll-off rates including $-12 \mathrm{~dB} /$ Octave, $-18 \mathrm{~dB} /$ Octave, and $-24 \mathrm{~dB} /$ Octave were investigated, employed and presented in literature [6-8].

In this research, a second-order Sallen-Key high-pass filters (Figure 3) and a fourth-order Sallen-Key high-pass filter (Figure 4) were studied and compared. The former has a slope of $-12 \mathrm{~dB} /$ Octave and a $20 \mathrm{~Hz}$ corner frequency whereas the latter has a slope of $-24 \mathrm{~dB} /$ Octave and the same corner frequency. Sallen-Key architecture is a widely used circuit topology for building second-order filters, also known as a voltage-controlled voltage source. The corner frequency and the passband gain of the second-order Sellen-Key high-pass filter are given by equation (3) and (4) respectively [9].

$$
\begin{aligned}
& f_{c}=\frac{1}{2 \pi \sqrt{R_{1} R_{2} C_{1} C_{2}}} \\
& G_{\text {pass }}=1+\frac{R_{4}}{R_{3}}
\end{aligned}
$$

where $f_{c}$ is the corner frequency and $G_{\text {pass }}$ is the passband gain.

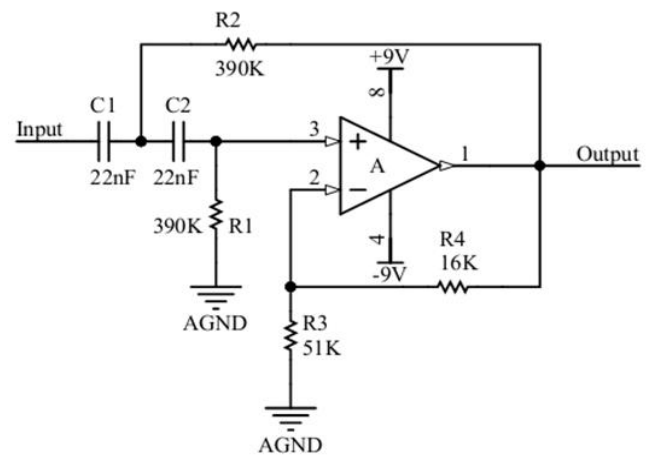

Fig. 3: The schematic of the second-order Sellen-Key high-pass active filter with a corner frequency of $20 \mathrm{~Hz}$ and a roll-off rate of $-12 \mathrm{~dB} /$ Octave

Figure 4 is the schematic of a fourth-order high-pass filter built by cascading two second-order Sallen-Key filters. The corner frequency of this fourth-order filter is determined based on the transfer functions detailed in reference [9].

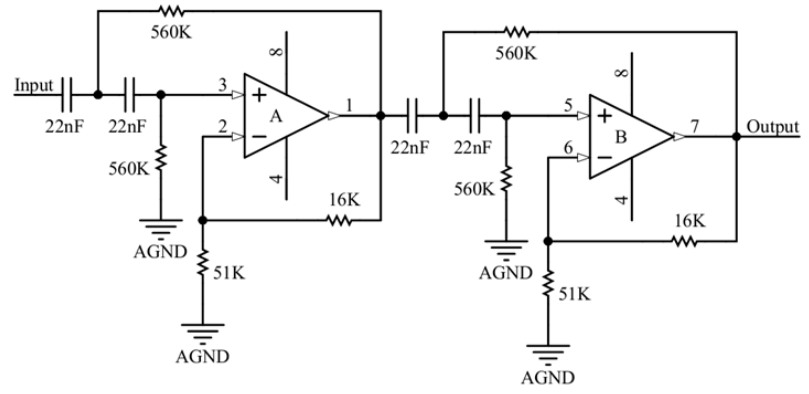

Fig. 4 The schematic of the fourth-order Sellen-Key high-pass active filter with a corner frequency of $20 \mathrm{~Hz}$ and a roll-off rate of $-24 \mathrm{~dB} /$ Octave

Since the noises coupled into the circuitry from electromagnetic radiation are mainly in the high frequency band, in this research, every amplification stage was followed by a secondorder Sallen-Key low-pass filter as shown in Figure 5. The corner frequency and the passband gain can also be calculated using equation (3) and (4) the same way as for high-pass filter design. In this research, the two second-order low-pass filters have the same properties and are placed before and after the second amplification stage as shown in Figure 1. The total frequency response of these two second-order low-pass filters should be equivalent to a low-pass filter cascaded by two second-order low-pass filters, namely a fourth-order low-pass filter. For this research, the corner frequency of the fourth-order low-pass filter is $500 \mathrm{~Hz}$ and the roll-off rate is $-24 \mathrm{~dB} /$ Octave [9].

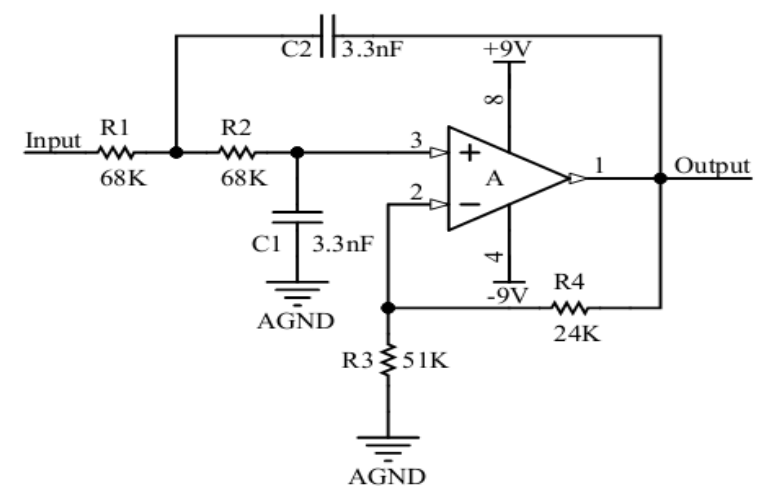

Fig. 5 The schematic of the second-order Sellen-Key low-pass active filter with a corner frequency of $709 \mathrm{~Hz}$ and a roll-off rate of $-12 \mathrm{~dB} /$ Octave

An issue related to low-pass filters is the selection of the sampling rate that is important in EMG signal acquisition. However it was not explicitly elucidated in EMG related literature. Collecting EMG signals from upper limbs is a typical example. Different sample rates, e.g., 400-500 Hz [10], 1000 $\mathrm{Hz}$ [11-13], $1024 \mathrm{~Hz}[14,15], 2000 \mathrm{~Hz}$ [16] and $4000 \mathrm{~Hz}$ [17] were used for converting the analog EMG signals to digital signals. Using a high sampling rate would reduce requirements on analog anti-aliasing filter but increase processing and computational load. According to the Nyquist-Shannon sampling theorem, when digitising a band-limited signal the sampling rate has to be two times higher than its band-limit otherwise aliasing may happen. Theoretically, as the highest useful frequency component of the EMG signals from an upper limb is less than $500 \mathrm{~Hz}$, a $1000 \mathrm{~Hz}$ sample rate is sufficient. However, the issue is more complicated than it looks. This is because the suitable sampling rate is also dependent on the rolloff rate of the anti-aliasing filter. The situation, where a sampling rate of $1000 \mathrm{~Hz}$ is sufficient for collecting the EMG signals with the maximum frequency less than $500 \mathrm{~Hz}$, can only be happened when an ideal low-pass anti-aliasing filter is used. In other words, the low-pass anti-aliasing filter must have an ideal property, that is, an infinite roll-off rate. In reality, this is impossible. As a result, the selection of the sampling rate has to consider the roll-off rate of the anti-aliasing filter, which is determined by the order of the low-pass filter. In this research, two low-pass filters with a corner frequency of $709 \mathrm{~Hz}$ and a slope of $-12 \mathrm{~dB} /$ Octave were cascaded to form a fourth-order low-pass filter with a $500 \mathrm{~Hz}$ corner frequency and a slope of $24 \mathrm{~dB} /$ Octave. The two low-pass filters are able to remove the noises above $500 \mathrm{~Hz}$. Meanwhile, the two low-pass filters also work as an anti-aliasing filter for analog-to-digital conversion [18]. As shown in Figure 6, the fourth-order low-pass filter (Line $\mathrm{C}$ ) has higher possibility to use a lower sampling rate $\mathrm{f}_{\mathrm{s} 1}$ 
than the second-order low-pass filter (Line D), by achieving the same attenuation to spectral replications at $500 \mathrm{~Hz}$ or even higher attenuation within $0-500 \mathrm{~Hz}$, without causing aliasing. In other words, if the same sampling rate $\mathrm{f}_{\mathrm{s} 2}$ is used, the fourthorder low-pass filter (Line E) can introduce lower amplitude of spectral replications on the spectral baseband $(0-500 \mathrm{~Hz})$ than the second-order low-pass filter (Line D). Figure 6 also reveals that, for the signals with the maximum frequency less than 500 $\mathrm{Hz}$ and using a Nyquist rate $\mathrm{A} / \mathrm{D}$ converter, a sampling rate of $1000 \mathrm{~Hz}$ may not provide the necessary aliasing rejection for the fourth-order low-pass filter (Line F). In this research, a sampling rate of $2000 \mathrm{~Hz}$ was chosen. The experiment showed the $2000 \mathrm{~Hz}$ sampling rate was higher enough to attenuate the frequencies above $500 \mathrm{~Hz}$. However, as the sampling rate gets higher the size of the data is increased. Therefore, in real applications, the selection of sampling rate higher than $2000 \mathrm{~Hz}$ may need to consider the hardware constrains.

Unlike conventional Nyquist rate A/D converters, Sigma-Delta $(\Sigma-\Delta)$ A/D converters use oversampling and decimation filtering technology. At the analog input end, a sampling rate that is much greater than the Nyquist rate is used. Through decimation and digital filtering, the sampling rate is reduced down to the Nyquist rate at digital output end. Sigma-Delta $(\Sigma-\Delta)$ conversion technology minimises the size of the data but reduces the requirements on the analog anti-aliasing filter simultaneously. It means that using a Sigma-Delta $(\Sigma-\Delta) \mathrm{A} / \mathrm{D}$ converter, the EMG signals can be recorded at an output sampling rate of 1000 samples per second (SPS).

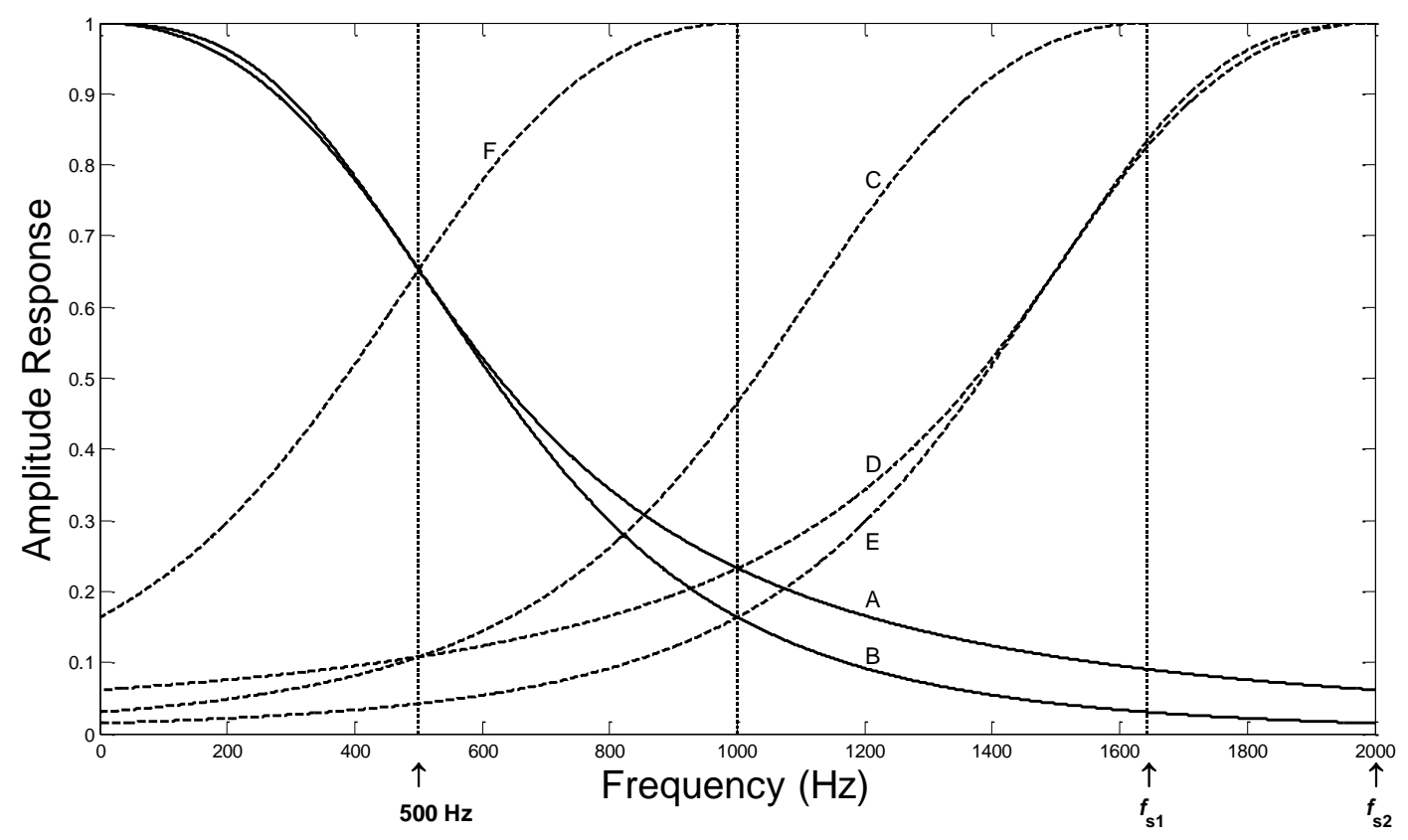

Fig. 6 The amplitude responses and spectral replications of Sellen-Key low-pass filters

Line A - the amplitude of the second-order low-pass filter

Line B - the amplitude of the fourth - order low-pass filter

Line $\mathrm{C}$ - the replication of the fourth-order low-pass filter at sampling rate of $\mathrm{f}_{\mathrm{s} 1}$

Line $\mathrm{D}$ - the replication of the second-order low-pass filter at sampling rate of $\mathrm{f}_{\mathrm{s} 2}$

Line $E$ - the replication of the fourth-order low-pass filter at sampling rate of $f_{\mathrm{s} 2}$

Line $\mathrm{F}$ - the replication of the fourth-order low-pass filter at sampling rate of $1 \mathrm{kHz}$

\section{EXPERIMENT}

To compare the effect of a fourth-order and a second-order high-pass filter on the low frequency components, the experiment circuit was configured as showed in Figure 7. After the electrodes and the pre-amplifier, the signals is conditioned using two different approaches before connecting to the SCB68 connector from National Instrument as illustrated in the block diagram in Figure 7, a hardware interface box for National Instrument PCI card. The difference between the two approaches is in the high-pass filter section. The first approach uses a second-order high-pass filter as showed in Figure 3 whereas the other is a fourth-order high-pass filter shown in Figure 4. The amplified and filtered EMG signals from the two approaches were simultaneously sampled and recorded using LabView. Hence, the EMG signals from the two approaches are actually from the same original source and in the same period of time but through different high-pass filters.
In the experiment, a data acquisition card PCI-6229 from National Instrument and LabView were used to collect EMG data. The amplified and filtered EMG signals were fed into a 68-pin shielded connector SCB-68 which is connected to the 16-Bit PCI-6229 DAQ card in a computer. A programme was developed in LabView to log the EMG data. Matlab was employed to analyse the spectra of the EMG signals. The EMG signals were recorded at a sampling rate of $2000 \mathrm{~Hz}$.

Three snap type pre-gelled surface EMG (sEMG) electrodes and a sEMG snap cable from Thought Technology Ltd were used. Two electrodes with the inter-spacing of $2 \mathrm{~cm}$ were placed on the forearm flexor carpi radialis muscle and the reference electrode was located on the wrist. Figure 8 illustrates the experiment configuration used in this research. 


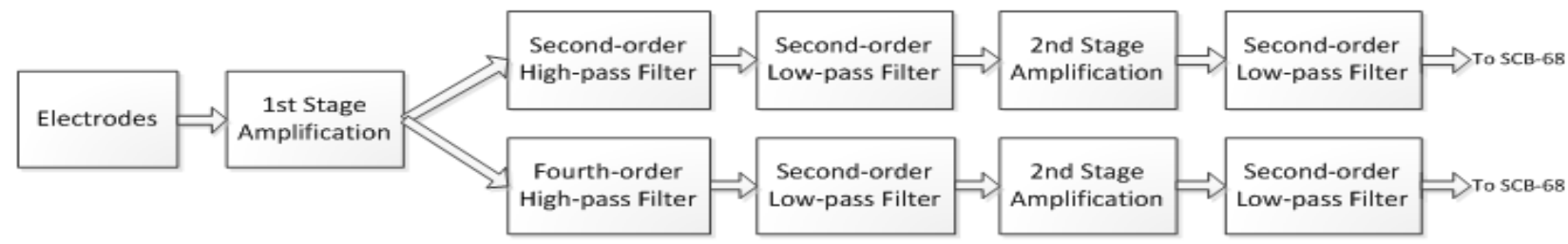

Fig. 7 Block diagram of the amplification and filtering circuit

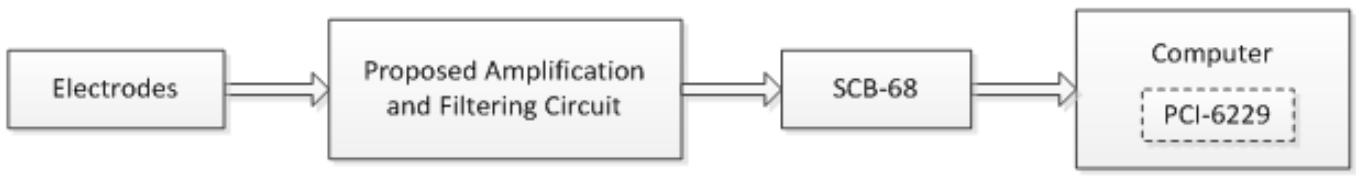

Fig. 8 The diagram of experiment configuration

The corner frequency of the high-pass and low-pass filter and the total gain of the amplification and filtering circuit shown in Figure 7 were set as follows:

- The high-pass corner frequency is $20 \mathrm{~Hz}$;

- The low-pass corner frequency is $500 \mathrm{~Hz}$;

- The total gain is set at about 2900 .

The human upper limb is able to perform sophisticated movements as it has multiple degrees of freedom. However, up until now, it is still a challenge for researchers to model and control human hand movement. In most cases, only certain hand gestures are studied. In this research, two upper limb movements were used to analyse and test the proposed amplification and filtering design. One is ball squeezing, which is the spherical grasping from the six basic hand grasping proposed by N. Fukaya [19] and the other is forearm flexion as illustrated in Figure 9.

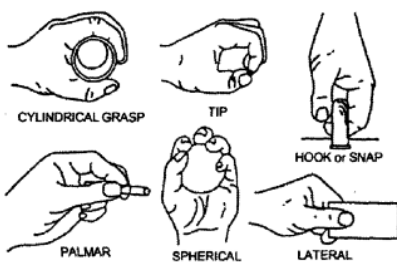

(a)

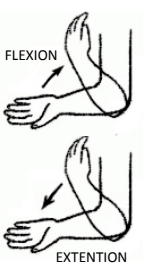

(b)
Fig. 9 (a) Six types of hand grasping and (b) Two forearm motions.

The spherical grasping is tested to squeeze a rubber ball and the forearm flexion to hold a bottle of water hung over the hand. For each hand movement, the test was repeated five times and collected five sets of data. Each test run had 2-second rest at the beginning and then followed the 2-second activation and 2second rest pattern. The experiment data were stored in datasheet files using LabView and then transferred to Matlab to conduct time-frequency analysis.

\section{DATA ANALYSIS}

Matlab was used to analyse experimental data and evaluate the proposed EMG amplification and filtering circuit. The EMG signal waveforms were displayed in Matlab. The activation segments of the two upper-limb movements, that is, when the target muscles were contracting, were clearly identified. Fourier transform was performed to the activation segments to analyse the spectrum.

\subsection{Ball Squeezing}

The activation segments from the five data sets for ball squeezing were analysed using Fourier transform. As shown in Figure 7, each test had two sets of data. One is the result of using a fourth-order high-pass filter while the other is the output from the second-order high-pass filter. Figure 9 and 10 is the comparison of the amplitude response of an activation segment randomly selected from the experiment activation segments between the two different approaches discussed in Section 4 .

(a)

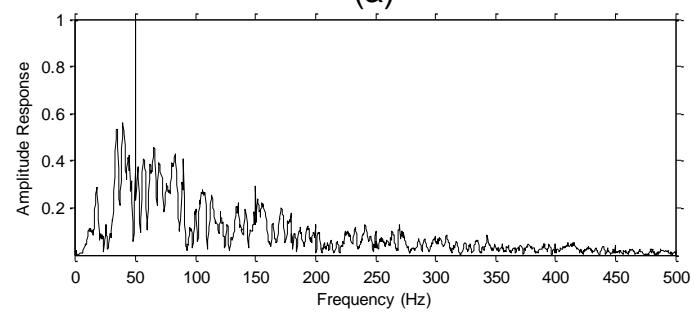

(b)

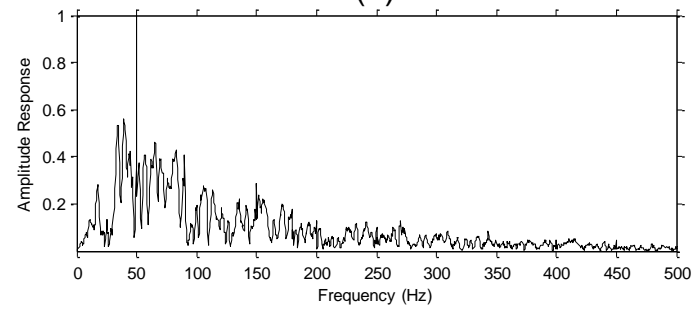

Fig. 9 The amplitude response of the EMG Signal of ball squeezing $(0-500 \mathrm{~Hz})$.

(a) Using the fourth-order high-pass filter (b) Using the second-order high-pass filter 
(a)

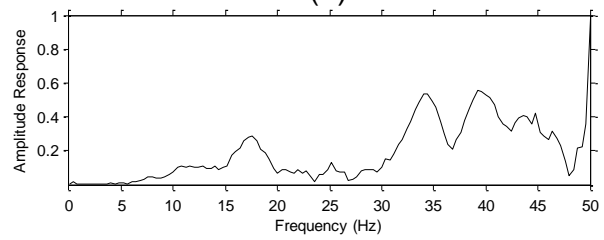

(b)

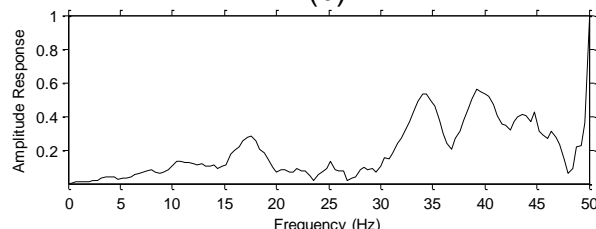

Fig. 10 The amplitude response of the EMG signal of ball squeezing (zoomed in 0-50 Hz).

(a) Using the fourth-order high-pass filter

(b) Using the second-order high-pass filter

\subsection{Forearm Flexion}

Similar to the movement of ball squeezing, the amplitude response of an activation segment of forearm flexion is presented in Figure 11 and 12.

(a)

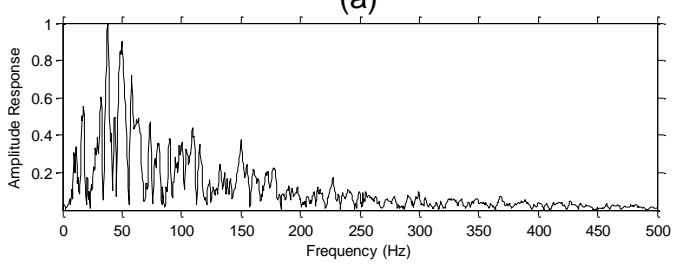

(b)

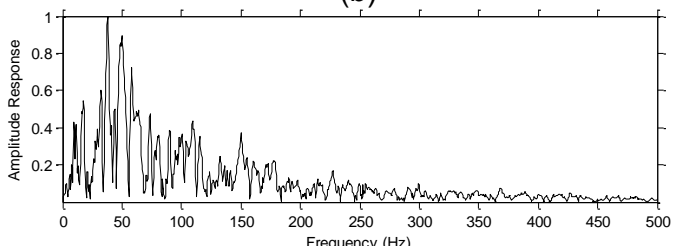

Fig. 11 The amplitude response of the EMG signal of forearm flexion $(0-500 \mathrm{~Hz})$.

(a) Using the fourth-order high-pass filter

(b) Using the second-order high-pass filter

(a)

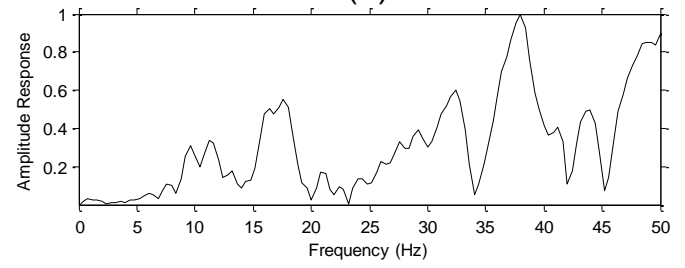

(b)

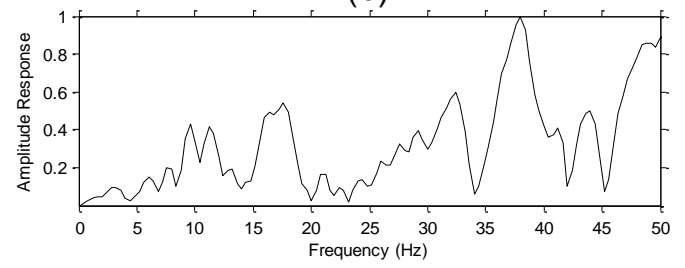

Fig. 12 The amplitude response of the EMG signal of forearm flexion (zoomed in 0-50 Hz).

(a) Using the fourth-order high-pass filter

(b) Using the second-order high-pass filter

As the results shown in Figure 9, 10, 11 and 12, there is only little improvement in terms of suppression to low frequency noises within the range of $0-15 \mathrm{~Hz}$, when the high-pass roll-off rate was doubled from $-12 \mathrm{~dB} /$ Octave to $-24 \mathrm{~dB} /$ Octave. A second-order high-pass filter could perform almost as good as a fourth-order high-pass filter in the tested EMG filtering circuitry.

\section{FINAL DESIGN}

Based on the above study and experiments, the final version of the proposed EMG amplification and filtering circuit consists of a pre-amplifier, a second-order high-pass filter, a second-order low-pass filter, an inverting amplifier, and a second-order lowpass filter. Figure 13 is the schematic of the circuit. The corresponding EMG signals collected from ball squeezing and forearm flexion movements using this circuit are presented in Figure 14.

The experiment also showed that, even when the limb keeps completely relaxed, the signal collected from the EMG circuit has some obvious noise signals. The frequency spectrum of the noise signals is displayed in Figure 15, which clearly reveals that the noise from the power line interferes with the $50 \mathrm{~Hz}$ component. This noise and its harmonics is the primary noise source. Conventionally, a notch filter at $50 \mathrm{~Hz}$ (or $60 \mathrm{~Hz}$ ) is employed to clean the power line noises. However, the majority energy of the EMG signal is within the $30-150 \mathrm{~Hz}$ range and there is no notch filter can perform to the ideal level to only eliminate $50 \mathrm{~Hz}$ (or $60 \mathrm{~Hz}$ ) noises without losing the neighbouring EMG signal components. As a result, the use of a notch filter suppresses the power line interference and at the same time, it causes the loss of useful information of the EMG signals. In addition, a notch filter at 50 (or 60) $\mathrm{Hz}$ is unable to attenuate its harmonics introduced by the nonlinearity of electronic components. Therefore, a notch filter is not recommended for EMG data acquisition circuitry [2, 3]. Various adaptive filter techniques have been proposed for noise cancellation [20-22] and have shown the potential for specific EMG signal processing.

\section{CONCLUSIONS}

This paper discusses the issues related to the design of surface EMG signal amplification and filtering circuits. The circuit design proposed was tested stage by stage and then integrated with a PC-based EMG data acquisition system through three electrodes. The system is able to successfully acquire the EMG signals and suppress the noises. The analysis of the experiment results validates the effectiveness of the proposed design. The comparison between the second-and fourth-order high-pass filter also confirms that a second-order high-pass filter has the potential to replace the fourth-order high-pass filter. Furthermore, the experiment also clearly shows that it is necessary to remove the noises from power line to increase signal-noise-ratio. 

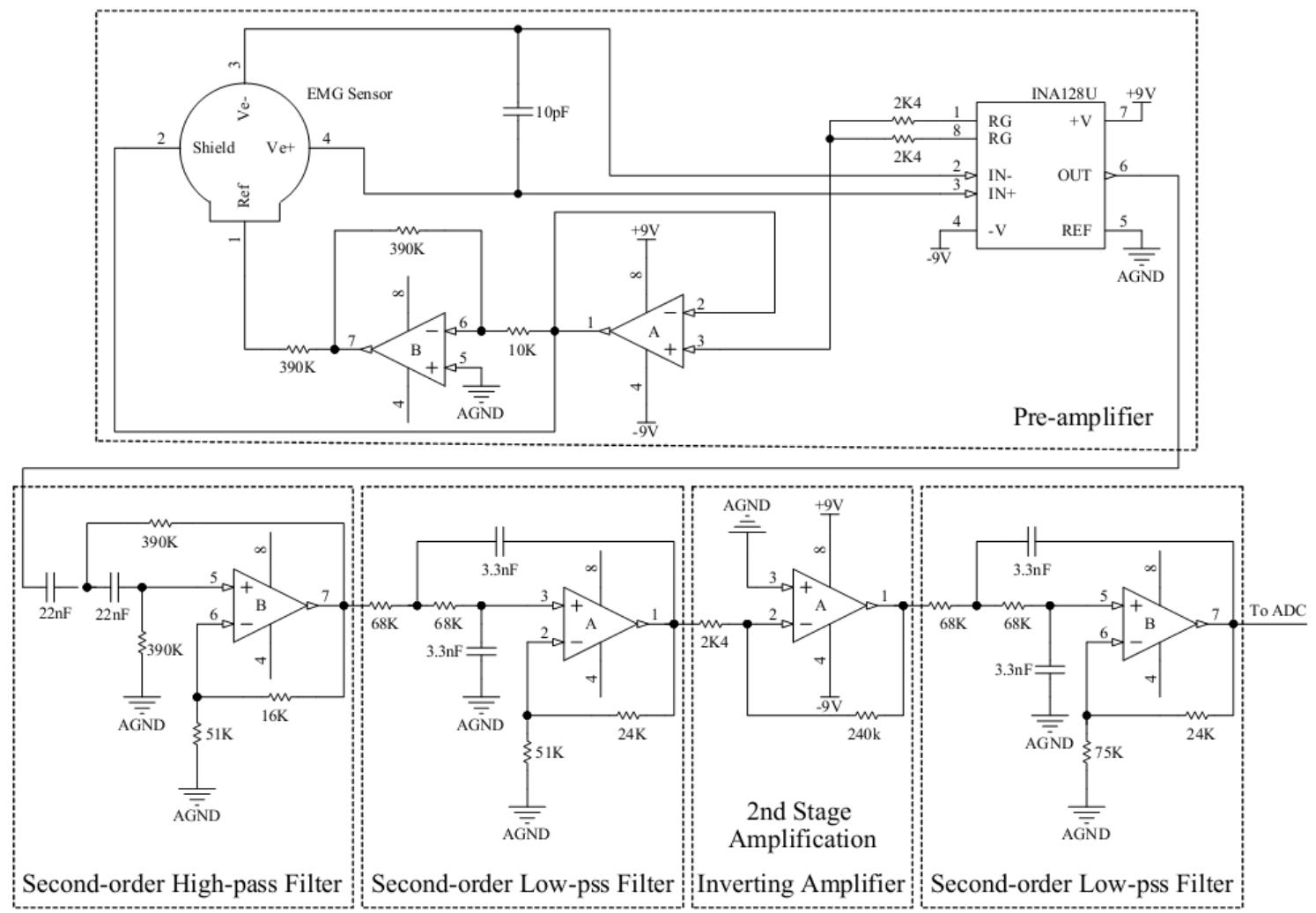

Fig. 13 The schematic of the proposed amplification and filtering circuit

(a)

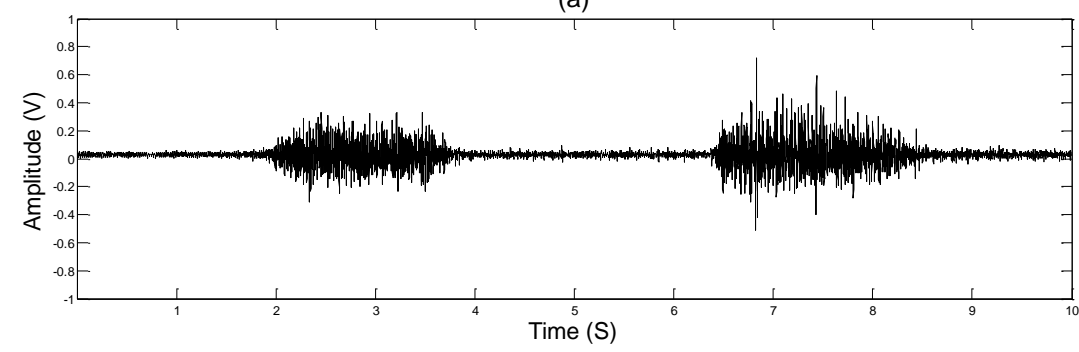

(b)

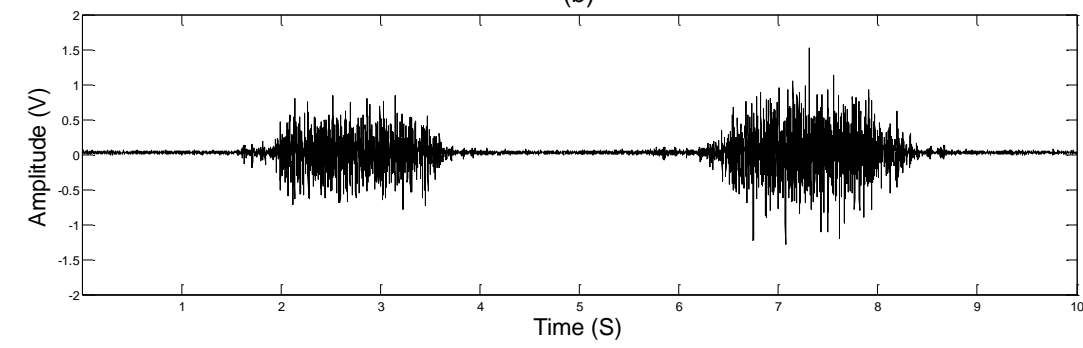

Fig. 14 The EMG signal waveforms in time domain.
(a) Ball Squeezing
(b) Forearm flexion

\section{ACKNOWLEDGMENTS}

This research is supported by the School of Engineering and Advanced Technology (SEAT), Massey University, New Zealand. A special thanks to the electronics technical team for the great assistance.

\section{REFERENCES}

[1] De Luca, C. J. 2006. Electromyography. Encyclopedia of Medical Devices and Instrumentation, pp. 98-109.

[2] De Luca, C. J. 2002. Surface electromyography: Detection and recording. DelSys Incorporated. 
[3] Day,S. 2002. Important factors in surface EMG Measurement. Calgary: Bortech Biomedical Ltd.

[4] INA128 Datasheet . 2005. Texas Instruments Incorporated.

[5] Zumbahlen, H. 2007. Phase Relations in Active Filters. Analog Dialogue, 41.

[6] Cosmanescu, A., Miller, B., Magno, T., Ahmed, A. and Kremenic, I. Design and Implementation of a Wireless (BluetoothÂA) Four Channel Bio-Instrumentation Amplifier and Digital Data Acquisition Device with UserSelectable Gain, Frequency, and Driven Reference. pp. 2053-2056.

[7] De Luca, C. J., Donald Gilmore, L., Kuznetsov, M. and Roy, S. H. 2010. Filtering the surface EMG signal: Movement artifact and baseline noise contamination. Journal of Biomechanics, vol. 43, no. 8, pp. 1573-1579.

[8] Smith, L. H., Hargrove, L. J., Lock, B. A. and Kuiken, T. A. 2011. Determining the Optimal Window Length for Pattern Recognition-Based Myoelectric Control: Balancing the Competing Effects of Classification Error and Controller Delay. Neural Systems and Rehabilitation Engineering, IEEE Transactions on, vol. 19, no. 2, pp. 186-192.

[9] Analysis of the Sallen-Key Architecture. 1999. Texas Instruments Incorporated.

[10] Guanglin, L., Yaonan, L., Zhiyong, Z., Yanjuan, G. and Rui, Z. Selection of sampling rate for EMG pattern recognition based prosthesis control. pp. 5058-5061.

[11] Cipriani, C., Antfolk, C., Controzzi, M., Lundborg, G., Rosen, B., Carrozza, M. C. and Sebelius, F. 2011. Online Myoelectric Control of a Dexterous Hand Prosthesis by Transradial Amputees. Neural Systems and Rehabilitation Engineering, IEEE Transactions on, vol. 19, no. 3, pp. 260-270.

[12] Hargrove, L. J., Scheme, E. J., Englehart, K. B. and Hudgins, B. S. 2010. Multiple Binary Classifications via Linear Discriminant Analysis for Improved Controllability of a Powered Prosthesis. Neural Systems and Rehabilitation Engineering, IEEE Transactions on, vol. 18, no. 1 , pp. 49-57.

[13] Oskoei, M. A. and Huosheng, H. 2008. Support Vector Machine-Based Classification Scheme for Myoelectric
Control Applied to Upper Limb. Biomedical Engineering, IEEE Transactions on, vol. 55, no. 8, pp. 1956-1965.

[14] Phinyomark, A., Nuidod, A., Phukpattaranont, P. and Limsakul, C. 2012. Feature extraction and reduction of wavelet transform coefficients for EMG pattern classification. Elektronika ir Elektrotechnika, vol. 122, no. 6, pp. 27-32.

[15] Jun-Uk, C., Inhyuk, M. and Mu-Seong, M. 2006. A RealTime EMG Pattern Recognition System Based on LinearNonlinear Feature Projection for a Multifunction Myoelectric Hand. Biomedical Engineering, IEEE Transactions on, vol. 53, no. 11, pp. 2232-2239.

[16] Zhang, X. and Zhou, P. 2012. Sample entropy analysis of surface EMG for improved muscle activity onset detection against spurious background spikes. Journal of Electromyography and Kinesiology, vol. 22, no. 6, pp. 901-907.

[17] Khushaba, R. N., Kodagoda, S., Takruri, M. and Dissanayake, G. 2012. Toward improved control of prosthetic fingers using surface electromyogram (EMG) signals. Expert Systems with Applications, vol. 39, no. 12, pp. 10731-10738.

[18] Hosking, R. H. 2012. Critical Techniques for High-Speed A/D Converters in Real-Time Systems.

[19] Fukaya, N., Toyama, S., Asfour, T. and Dillmann, R Design of the TUAT/Karlsruhe humanoid hand. pp. 17541759.

[20] Yacoub, S., Raoof, K. and Eleuch, H. Filtering of Cardiac and Power Line in Surface Respiratory EMG Signal.

[21] Golabbakhsh, M., Masoumzadeh, M. and Sabahi, M. F. 2011. ECG and power line noise removal from respiratory EMG signal using adaptive filters. Majlesi Journal of Electrical Engineering, vol. 5, no. 4.

[22] A. B. Sankar, D. Kumar, and K. Seethalakshmi, "Performance study of various adaptive filter algorithms for noise cancellation in respiratory signals," Signal Processing: An International Journal (SPIJ), vol. 4, no. 5, pp. 267, 2010.

[23] De Luca, C. J., Kuznetsov, M. L., Gilmore, D. and Roy, S. H. 2012. Inter-electrode spacing of surface EMG sensors: Reduction of crosstalk contamination during voluntary contractions," Journal of Biomechanics, vol. 45, no. 3, pp. 555-561. 